\title{
Capture barrier distributions: some insights and details
}

\author{
N. Rowley \\ Institut Pluridisciplinaire Hubert Curien (UMR 7178: CNRS/ULP), \\ 23 rue du Loess, F-67037 Strasbourg Cedex 2, France \\ N. Grar \\ Department of Physics, University of Sétif, Algeria \\ M. Trotta \\ INFN, Sezione di Napoli, I-80126, Italy
}

(Dated: September 28, 2007)

\begin{abstract}
The 'experimental barrier distribution' provides a parameter-free representation of experimental heavy-ion capture cross sections that highlights the effects of entrance-channel couplings. Its relation to the $s$-wave transmission is discussed, and in particular it is shown how the full capture cross section can be generated from an $l=0$ coupled-channels calculation. Furthermore, it is shown how this transmission can be simply exploited in calculations of quasi-fission and evaporation-residue cross sections. The system ${ }^{48} \mathrm{Ca}+{ }^{154} \mathrm{Sm}$ is studied in detail. A calculation of the compound-nucleus spin distribution shows up a possible energy dependence of barrier weights due to polarization arising from target and projectile quadrupole phonon states; an effect which also gives rise to an entrance-channel 'extra-push'.
\end{abstract}

PACS numbers: 24.10.-i, 24.10.Eq, 24.60.Dr, 25.60.Pj, 25.70.Gh, 25.70.-z, 25.70.Jj

\section{INTRODUCTION}

The complexity of heavy-ion reactions depends to a large extent on the charge product $Z_{1} Z_{2}$ of the colliding nuclei. However, for all but the very highest- $Z_{1} Z_{2}$ reactions, the first stage in the creation of a composite system is determined by the crossing of an external Coulomb barrier, or in the case of strong entrance-channel couplings, a "distribution of barriers". This stage of the reaction is referred to as "capture" or sometimes "barrier-crossing". For very heavy systems such as $\mathrm{Pb}+\mathrm{Pb}$, this will clearly not be true, since there will be a significant overlap of the nuclear densities before the Coulomb barrier is reached. This will lead to strong dissipative effects and an important flow of nucleons between the colliding nuclei. However, recent experiments have shown $[1,2]$ that even for systems of the type leading to superheavy-element creation by cold fusion, the concept of a distribution of external Coulomb barriers is still valid. The results of this paper should apply to any collision where this is the case.

For light and intermediate-mass reactions, the composite system will evolve to form an equilibrated, compact compound nucleus $(\mathrm{CN})$. This we refer to as "fusion". The $\mathrm{CN}$ will then cool by the emission of light particles (neutrons, protons and $\alpha$-particles) to create longlived evaporation residues (ER). For heavier, composite systems an increasing fraction of the capture cross section $\sigma_{\text {cap }}$ will undergo quasi-fission $(\mathrm{QF})$ before $\mathrm{CN}$ formation. Furthermore the $\mathrm{CN}$ itself may fission (fusionfission; FF). Thus the experimental difficulties in measuring $\sigma_{\text {cap }}$ increase with $Z_{1} Z_{2}$; for this reason, the experiments $[1,2]$ mentioned above determined the capture barrier distribution from the large-angle quasi-elastic scattering [3]. In the present paper, we wish to discuss the properties of the capture cross section and the extent to which it can be represented as a distribution of barriers. The consequences of this on other cross sections will also be explored.

The "experimental barrier distribution" was introduced in Ref. [4] as

$$
D(E) \equiv \frac{1}{\pi R^{2}} \frac{d^{2}\left(E \sigma_{\mathrm{cap}}\right)}{d E^{2}},
$$

where $E$ is the center-of-mass energy and $R$ is some average barrier radius chosen simply to normalize the area of $D(E)$ to unity. (Note that this was referred to as the "fusion" barrier distribution, since fusion and capture were identical in the systems originally discussed. Here we shall refer to it as the "capture" barrier distribution, or simply the barrier distribution.) For the classical capture cross section from a single barrier, Eq. (1) gives $[4,5]$

$$
D_{\text {class }}(E)=\delta(E-B),
$$

where $B$ is the barrier height. In the single-barrier quantum mechanical problem [5], $D$ is more generally a function having unit area strongly peaked at $E=B$.

The quantity $D(E)$ frequently possesses detailed structures [5] that reflect the presence of different barriers generated by coupling to the collective excitations of the target and projectile in the entrance channel. Transfer channels may also play an important role [6]. It can also be shown $[7,8]$ that the first derivate of $E \sigma_{\text {cap }}$ has a physical interpretation in terms of the total $s$-wave transmission in the entrance channel

$$
T_{0}^{\mathrm{tot}} \approx \frac{1}{\pi R^{2}} \frac{d\left(E \sigma_{\mathrm{cap}}\right)}{d E} .
$$


can be rather well obtained from such a calculation.

\section{RELATING $T_{0}^{\text {tot }}, \sigma$ AND $D$}

\section{A. Single barrier}

Henceforth we shall simply use the symbol $\sigma$ for the capture cross section. The symbol $\sigma_{l}$ will refer to the partial capture cross section for a given $l$ value (particularly $\sigma_{0}$ for the $s$-wave). Other physical cross sections will take an appropriate suffix.

For a single barrier we may write the s-wave transmission coefficient very generally as

$$
T_{0} \equiv T_{0}(E-B) \text {. }
$$

For a system of reduced mass $m$, the partial cross section for $l=0$ is related to $T_{0}$ by

$$
E \sigma_{0}=\left(\frac{\pi \hbar^{2}}{2 m}\right) T_{0}(E-B)
$$

and the total cross section given by

$$
E \sigma=\left(\frac{\pi \hbar^{2}}{2 m}\right) \sum_{l=0}^{\infty}(2 l+1) T_{l}(E-B) .
$$

We may now follow the steps of Wong [14], used to derive the total cross section from the transmission through a parabolic barrier. Note, however, that we make no assumption concerning the particular form of the transmission function $T$ which will depend on the shape of the potential.

First we replace $T_{l}$ by $T_{0}\left(E^{\prime}-B\right)$, with

$$
E^{\prime}=E-\frac{l(l+1) \hbar^{2}}{2 m R^{2}} .
$$

That is, we approximate the centrifugal potential by its value at the barrier radius $R$. Thus

$$
E \sigma \approx\left(\frac{\pi \hbar^{2}}{2 m}\right) \sum_{l=0}^{\infty}(2 l+1) T_{0}\left(E^{\prime}-B\right) .
$$

Replacing the sum by an integral we have

$$
E \sigma \approx\left(\frac{\pi \hbar^{2}}{2 m}\right) \int_{0}^{\infty}(2 l+1) T_{0}\left(E^{\prime}-B\right) d l
$$

and using the expression (8) for $E^{\prime}$ we may write the rather general relation between the s-wave transmission and the total cross section $\sigma$

$$
E \sigma \approx \pi R^{2} \int_{0}^{E} T_{0}\left(E^{\prime}-B\right) d E^{\prime}
$$

where the radius $R$ is taken to have its s-wave value but no reference has been made to the particular shape of the
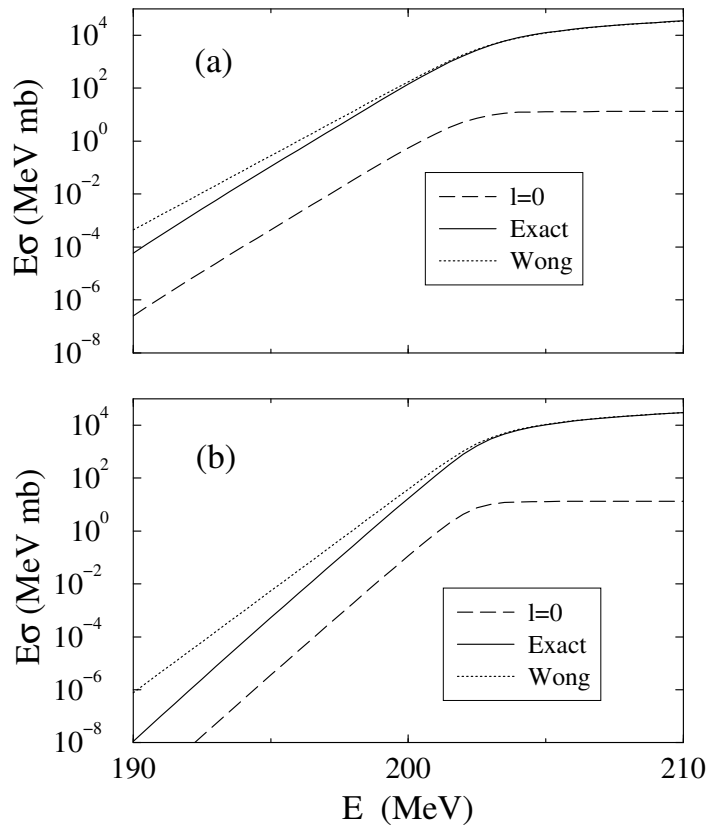

FIG. 2: For ${ }^{100} \mathrm{Mo}+{ }^{100} \mathrm{Mo}$ and a single barrier with $B=$ $202.4 \mathrm{MeV}$, we show $E \sigma_{0}$ and $E \sigma$, where $\sigma_{0}$ and $\sigma$ are the exact optical-model s-wave partial cross section (dashed curve) and total capture cross section (solid curve) respectively. The results of Eq. (11) are practically indistinguishable from the exact results on this scale: (a) corresponds to a surface diffuseness $a=0.6 \mathrm{fm}$ and (b) to $a=1.2 \mathrm{fm}$. Note the significant deviations in the Wong cross sections below the barrier (dotted curves) using a curvature calculated at the barrier top.

barrier. In particular, the barrier "curvature" $\hbar \omega$ does not occur explicitly in this equation.

If, however, we use the Hill-Wheeler approximation for a parabolic barrier with this curvature,

$$
T_{0}=\frac{1}{1+\exp (2 \pi[B-E] / \hbar \omega)},
$$

then Eq. (11) yields the well known Wong cross section $[14]$

$$
E \sigma=\frac{1}{2} \hbar \omega R^{2} \ln (1+\exp (2 \pi[E-B] / \hbar \omega)) .
$$

Eq. (11) should, however, be more generally applicable and we shall first test it with an optical-model calculation.

Fig. 2 shows the results of (uncoupled) optical-model calculations with a real potential which is essentially exponential in the tail and having a diffusenesses of (a) $a=0.6 \mathrm{fm}$ and (b) $a=1.2 \mathrm{fm}$. In both cases the barrier height is $B=202.4 \mathrm{MeV}$ and the Coulomb potential corresponds to ${ }^{100} \mathrm{Mo}+{ }^{100} \mathrm{Mo}$. The imaginary potential is confined to the nuclear interior to simulate a pure ingoing-wave boundary condition. The dashed curves show the $E \sigma_{0}$ obtained by a solution of the Schroedinger equation. The solid curve shows the corresponding $E \sigma$. 


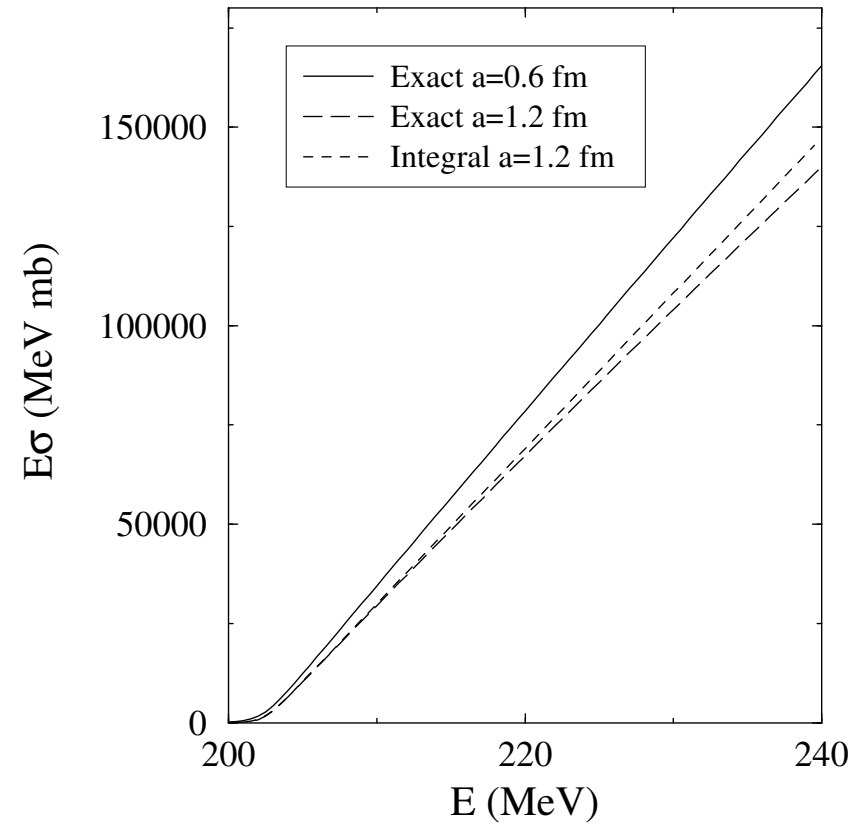

FIG. 3: The same quantities as in Fig. 2 on a linear scale and at higher $E$. For $a=0.6 \mathrm{fm}$ the integral (11) is almost indistinguishable from the exact results and is not shown. For $a=1.2 \mathrm{fm}$ there are small differences due to the fact that the barrier for high $l$ values shifts to smaller radii. In this respect, the Wong cross section gives a very similar result to the integral.

(Throughout this paper, cross sections will be represented as $E \sigma$ in units of $\mathrm{MeV}$ mb.) The cross section derived from Eq. (11) is indistinguishable from the exact result on this scale. The dotted curves show the Wong cross section (13). One can see that the cross section falls more rapidly for the larger diffuseness and that the discrepancies between Wong and the exact calculation become very large at deep sub-barrier energies [15]. This discrepancy is probably best quantified by the logarithmic derivative of the low-E cross section (see Sect. III D). In both cases, however, the relation (11) gives excellent results. The integration in that equation was performed using Simpson's rule. We use an integration step of $0.2 \mathrm{MeV}$ throughout the paper.

Thus we have reduced the calculation of a full capture cross section to solving the Schroedinger equation for a single $l$ value. This is not a particularly important achievement for a simple optical-model calculation but it could be of enormous benefit if it can be extended to coupled-channels calculations, where the time taken for each $l$ value may be relatively long, and the calculation may become numerically unstable for large angular momentum values.

Fig. 3 shows $E \sigma$ for the two values of diffuseness on a linear scale at energies well above the barrier. For $a=0.6 \mathrm{fm}$ the integral is still almost indistinguishable on this scale from the exact results. For the larger diffuseness, the curve has a smaller slope since the bar- rier radius must be smaller if we are to maintain the same barrier height of $202.4 \mathrm{MeV}\left(R_{0.6}=11.91 \mathrm{fm}\right.$ and $R_{1.2}=10.72 \mathrm{fm}$; see Eq. (22)). Now a small discrepancy shows up between the integral and the exact results since the barrier position is $l$-dependent and this dependence increases with increasing diffuseness [16]. We note that the s-wave barrier is $a$-dependent and that this essentially gives a first-order correction to the cross section. This is correctly accounted for by the above formalism. In addition, however, there is a second-order effect which depends on $l$ as well as the diffuseness. This is not accounted for, and we shall not pursue this further in the present paper.

\section{B. Several barriers: variation of the barrier radius}

Eq. (4) implies that we can write a similar weighted sum for the total s-wave transmission $T_{0}^{\text {tot }}$

$$
T_{0}^{\mathrm{tot}}(E)=\sum_{\alpha} w_{\alpha} T_{0}\left(E-B_{\alpha}\right)
$$

Following the above derivation of Eq. (11), we now find

$$
E \sigma \approx \pi \sum_{\alpha} \int_{0}^{E} R_{\alpha}^{2} w_{\alpha} T_{0}\left(E^{\prime}-B_{\alpha}\right) d E^{\prime}
$$

and if one sets all the $R_{\alpha}$ equal to a common value $R$, one recovers Eq. (11), which now relates the total capture cross section (summed over all $l$ ) to the total s-wave transmission (summed over all barriers):

$$
E \sigma \approx \pi R^{2} \int_{0}^{E} T_{0}^{\mathrm{tot}}\left(E^{\prime}\right) d E^{\prime}
$$

For small deformations of target and projectile, the above approximation of a fixed $R$ is reasonable. However, for large deformations, there may be relatively large differences in the positions of the different Coulomb barriers and this may lead to some errors in extracting the total cross section from a calculation with $l=0$. We can, however, circumvent this problem by noting that the derivative of the s-wave transmission coefficient is strongly peaked at $E=B$ for each barrier. Thus if we have an analytic expression for $R_{\alpha} \equiv R\left(B_{\alpha}\right)$ we may write

$$
\frac{d}{d E^{\prime}} \sum_{\alpha} w_{\alpha} R_{\alpha}^{2} T_{0}^{\alpha}\left(E^{\prime}\right) \approx R^{2}\left(E^{\prime}\right) \frac{d T_{0}^{\mathrm{tot}}\left(E^{\prime}\right)}{d E^{\prime}}
$$

with $T_{0}^{\alpha}\left(E^{\prime}\right) \equiv T_{0}\left(E^{\prime}-B_{\alpha}\right)$. Thus the integrand of Eq. (15) may be written

$$
\sum_{\alpha} w_{\alpha} R_{\alpha}^{2} T_{0}^{\alpha}\left(E^{\prime}\right) \approx \int_{0}^{E^{\prime}} R^{2}(B) \frac{d T_{0}^{\mathrm{tot}}(B)}{d B} d B
$$

where we use the integration variable $B$ to emphasize the fact that this integral is equivalent to the sum over the 
barriers $\alpha$. Finally this gives

$$
E \sigma \approx \pi \int_{0}^{E} \int_{0}^{E^{\prime}} R^{2}(B) \frac{d T_{0}^{\mathrm{tot}}(B)}{d B} d B d E^{\prime}
$$

and we have again achieved our aim of expressing the total capture cross section for all $l$ in terms of that simply for $l=0$. The integral over $E^{\prime}$ is equivalent to the sum over $l$ as in Eq. (11).

In both expressions (16) and (19), all of the information on the channel couplings (that is, on the barrier distribution $\left.\left(w_{\alpha}, B_{\alpha}\right)\right)$ is contained in the single function $T_{0}^{\text {tot}}$, which can be obtained from a single calculation with $l=0$. We have passed from the single integral of Eq. (16) to the double integral (19) in order account for the variation of $R_{\alpha}$ for the different barriers, since $d T_{0} / d E$ is peaked for each barrier. However, more importantly, this will also allow us to introduce into the integrand of Eq. (19) any other function of the barrier position $B$. In particular we shall later introduce the possibility of a quasi-fission component of the reaction which is barrier dependent due to the "compactness" [17] of the configuration at the barrier.

Since the Coulomb barrier will almost always occur in the region where the nuclear potential is approximately exponential, it is relatively easy to obtain an expression for $R(B)$. At the barrier $V_{\mathrm{C}}^{\prime}+V_{\mathrm{N}}^{\prime}=0$, and since $V_{\mathrm{N}}$ is exponential,

$$
V_{\mathrm{N}}(R)=-a \frac{Z_{1} Z_{2} e^{2}}{4 \pi \varepsilon_{0} R^{2}} \equiv-a \frac{Z}{R^{2}}
$$

and thus $V_{\mathrm{C}}^{\prime}+V_{\mathrm{N}}^{\prime}=0$ yields

$$
B=\frac{Z}{R}\left(1-\frac{a}{R}\right)
$$

Solving this quadratic equation for $R$ we obtain

$$
R(B)=\frac{Z}{2 B}\left(1+\left[1-\frac{4 a B}{Z}\right]^{1 / 2}\right)
$$

For small $a$, the approximation $R(B)=Z / B-a$ may be adequate, but in mapping from an $l=0$ calculation to the full cross section, we shall use the more exact relation (22) in the double integral of Eq. (19).

Of course we have again achieved little in the case where we have an expression in the form of Eq. (14) and know the values the the barrier heights $B_{\alpha}$ and their weights $w_{\alpha}$. However, Eq. (19) contains no reference to these, which are in any case not a natural output of a coupled-channels calculation. Indeed the very existence of eigenchannels assumed in Eqs. $(4,14)$ can be proved only under the very restricted conditions mentioned in the introduction. However, we note that in Eq. (19) there is no reference to eigenchannels, or weights, or barrier heights, and we may hope that it will, therefore, apply more generally to results from coupled-channels calculations.

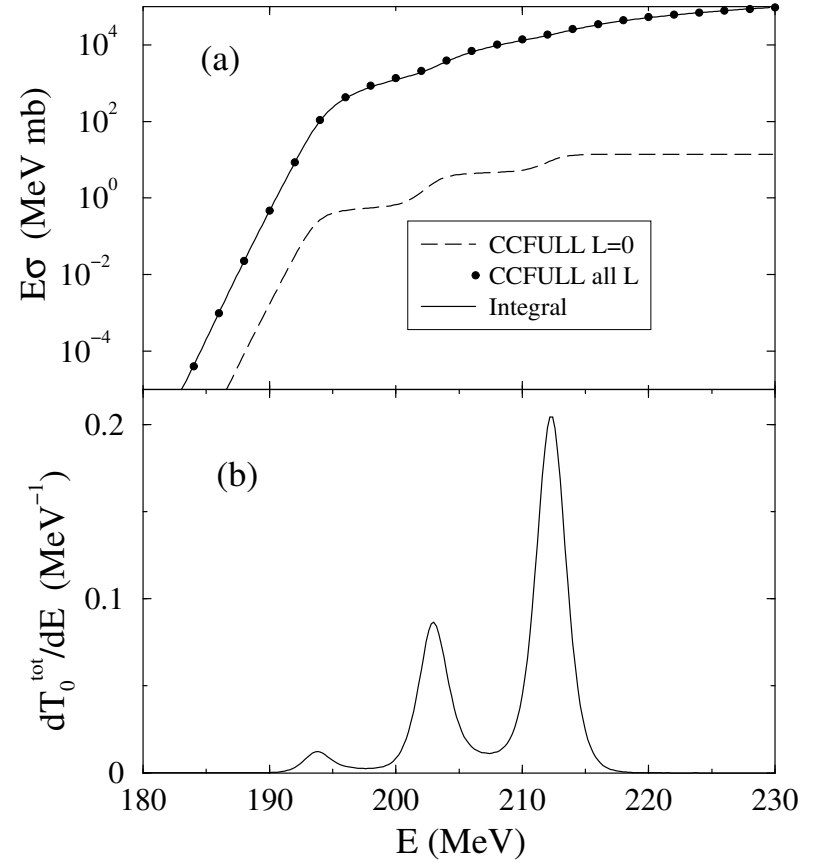

FIG. 4: (a) For ${ }^{100} \mathrm{Mo}+{ }^{100} \mathrm{Mo}$ with coupling to the first quadrupole-phonon state in each nucleus [1,1], we show $E \sigma_{0}$ (dashed curve) and $E \sigma$ (circles) both calculated with CCFULL. The results of Eq. (19) are shown by the solid line. (b) Shows the corresponding 'barrier distribution' $d T_{0}^{\text {tot }} / d E$.

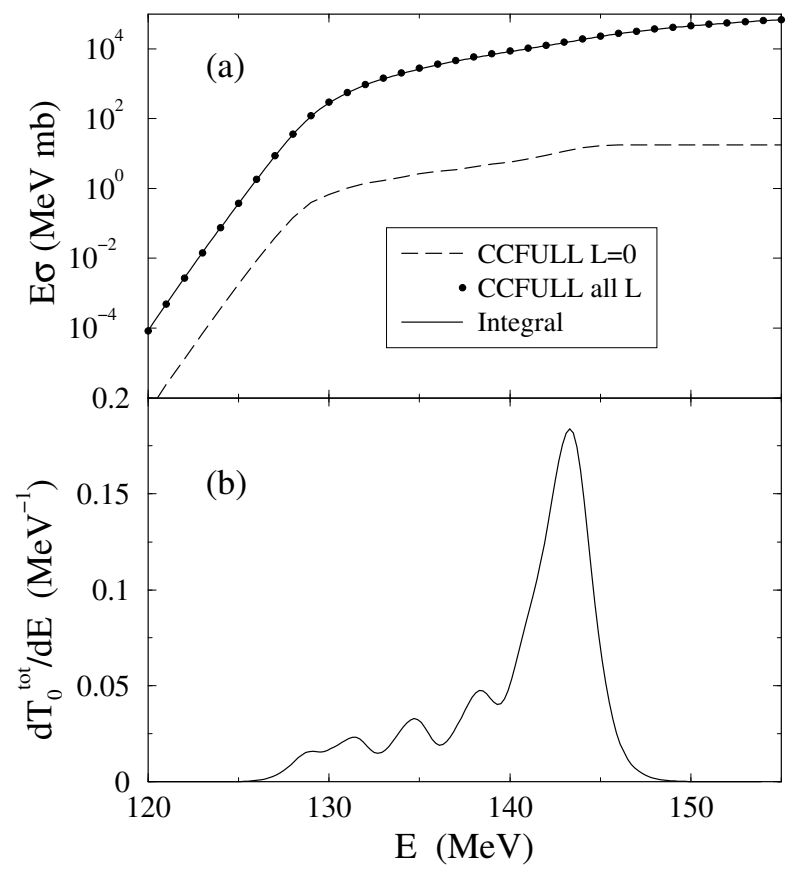

FIG. 5: Same quantities as in Fig. 4 but for the system ${ }^{48} \mathrm{Ca}$ $+{ }^{154} \mathrm{Sm}$ with quadrupole couplings to the first six excited states $[0,6]$ of the ${ }^{154} \mathrm{Sm}$ ground-state rotational band $\left(0^{+}\right.$to $\left.12^{+}\right)$.

Throughout the rest of this paper we shall present a 
number of coupled-channels calculations to demonstrate our results. These will all use a nuclear potential which is essentially exponential in the barrier region with a surface diffuseness $a=0.6 \mathrm{fm}$. The potential is, therefore, uniquely specified by quoting the barrier height $B_{\mathrm{nc}}$ with no coupling. For the channel couplings, we shall take throughout a coupling radius $r_{0}=1.20 \mathrm{fm}$. Couplings are, therefore, completely specified by the excitation energies $E_{I^{\pi}}$ of the states concerned (spin $I$ and parity $\pi$ ), the corresponding deformation parameters of the states $\beta_{L}$, and by the number of excited states (rotational or vibrational) included in the calculations. These are denoted $\left[N_{\text {projectile }}, N_{\text {target }}\right]$. Thus an uncoupled calculation would be denoted $[0,0]$.

It can be seen in Figs. 4, 5 that the expression (19) works extremely well. Fig. 4 shows results for the vibrational system ${ }^{100} \mathrm{Mo}+{ }^{100} \mathrm{Mo}$ with coupling to the first quadrupole-phonon state $\left(E_{2+}=0.53 \mathrm{MeV} ; \beta_{2}=0.23\right)$ in each nucleus and $B_{\mathrm{nc}}=202.4 \mathrm{MeV}$. (This [1,1] coupling scheme is a truncation of the more physical couplings used in Ref. [12], and whose results are shown in Fig. 1. This clearly will not fit the experimental data but the fact that it gives rise to discrete barriers will facilitate some of our later discussions.) The dashed curve in part (a) of the figure shows $E \sigma_{0}$ calculated using the program CCFULL [18]. The open circles show the full $E \sigma$ calculated using the same program but including up to $l=100$. The solid curve shows the $E \sigma$ generated from the s-wave calculation using Eq.(19), and can be seen to give excellent results. Part (b) of the figure shows the 'barrier distribution' defined as $d T_{0}^{\text {tot }} / d E$ (see next subsection III C).

Fig. 5 shows all the same quantities as Fig. 4 but now for the system ${ }^{48} \mathrm{Ca}+{ }^{154} \mathrm{Sm}$ with quadrupole coupling $\left(\beta_{2}=0.30, \beta_{4}=0.05\right)$ to the first six excited rotational states of the ${ }^{154} \mathrm{Sm}$ ground-state rotational band (that is up to the $12^{+}$state) and an inert ${ }^{48} \mathrm{Ca}$ (coupling $[0,6]$ ). The $2^{+}$state has energy $82 \mathrm{keV}$ and the other energies were taken to follow an $I(I+1)$ law. Eq. (19) is again seen to give excellent results. This system is discussed in more detail in Sect. IV.

For very heavy ions, one may have to consider hundreds of partial waves to obtain convergence of the capture cross section and some problems of stability of the CC calculations may arise, since for the higher partial waves the energy in question may be very far below the total potential barrier. Furthermore, the performance of coupled-channels calculations becomes time consuming for many channels and reducing this to a single calculation for $l=0$ has very obvious benefits. The calculation of the double integral in Eq. (19) is very rapid, even compared with solving the coupled equations for just a single $l$. This is especially important when trying to optimize parameters to fit experimental data. Note also that we need to calculate only up to energies where $d T_{0}^{\text {tot }} / d E$ becomes 0 , even if we require cross sections above this energy.

\section{Defining the barrier distribution}

The most natural mathematical definition of the barrier distribution is $d T_{0}^{\text {tot }} / d E$ since for a single barrier it gives a function normalized to 1 and peaked at $E=B$. Thus for many barriers we obtain a sum of functions each having a weight $w_{\alpha}$ and peaked at $B_{\alpha}$. From Eq. (19) we see that this is equal to

$$
D(E)=\frac{d}{d E} T_{0}^{\mathrm{tot}}(E)=\frac{1}{\pi R^{2}(E)} \frac{d^{2}(E \sigma)}{d E^{2}} .
$$

Apart from the $E$-dependence of $R$ (actually a $B$ dependence), this is of course the usual definition [4] of the 'experimental barrier distribution' (1).

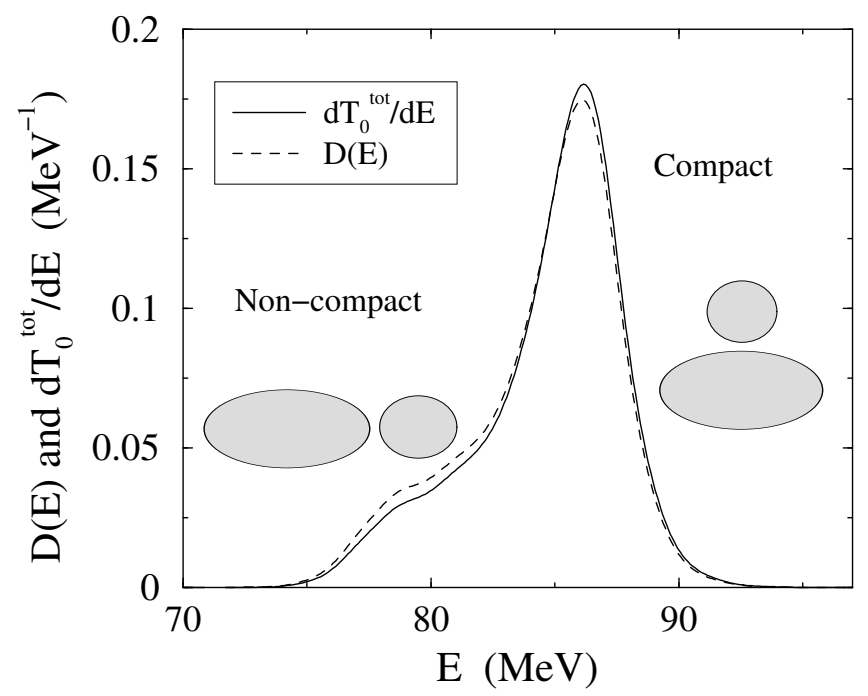

FIG. 6: For the system ${ }^{16} \mathrm{O}+{ }^{238} \mathrm{U}$ we show the difference between including the barrier-dependence of the radius and ignoring it (see Eq. (24)). Both curves are normalized to unity. The insets show schematically how the high barriers correspond to a more 'compact' configuration than the low ones (see Sect. IV B).

We do not, however, suggest the inclusion of such an energy dependence in the experimental results, since this involves introducing an unknown theoretical parameter $a$. Dividing the second derivative by a fixed $\pi R^{2}$ merely introduces a harmless overall normalization.

Of course whatever one does to the data should also been done to a calculation before a comparison is made. So the second derivative of the theoretical $E \sigma$ of Eq. (19) should be treated in the same way as its experimental equivalent. However, in this paper we wish to advocate simplified CC calculations using only $l=0$. In that case we simply note that

$$
\frac{d^{2}(E \sigma)}{d E^{2}}=\pi R^{2}(E) \frac{d T_{0}^{\mathrm{tot}}(E)}{d E} .
$$

Now the barrier-dependence of $R$ can be simply included since the value of the diffuseness used in the calculations is known. 
Fig. 6 shows the difference between $d T_{0}^{\text {tot }} / d E$ and the usual 'experimental barrier distribution' of Eq. (1), with $R$ chosen so that $D(E)$ is also normalized to unity for the system ${ }^{16} \mathrm{O}+{ }^{238} \mathrm{U}$ (calculated with $E_{2^{+}}=45 \mathrm{keV}$, $\left.\beta_{2}=0.275, \beta_{4}=0.05\right)$. The differences are seen to be relatively small, which means that for most purposes, the single integral of Eq. (16) will give good results. However, a major advantage of the double integral of Eq. (19) is that we can also introduce into it other functions of $B$. We shall demonstrate this in Sect. IV where we shall introduce quasi-fission through the notion of 'compactness' [17].

\section{Logarithmic derivative}

It has been noted recently that at deep sub-barrier energies, many heavy-ion fusion cross sections fall off anomalously rapidly (see for example, Refs. [19-21]). This phenomenon is perhaps best displayed through the logarithmic derivative of the cross section which becomes slowly varying at these energies, at values consistent with a greater surface diffuseness than considered normal. For example, Dasgupta [21] shows that in the system ${ }^{16} \mathrm{O}+$ ${ }^{208} \mathrm{~Pb}$, the experimental $d \ln (E \sigma) / d E$ is consistent with a value of $a=1.65 \mathrm{fm}$. However, the behavior of the cross section at high energies appears to require a different value of $a$ (c.f. Fig. 3).

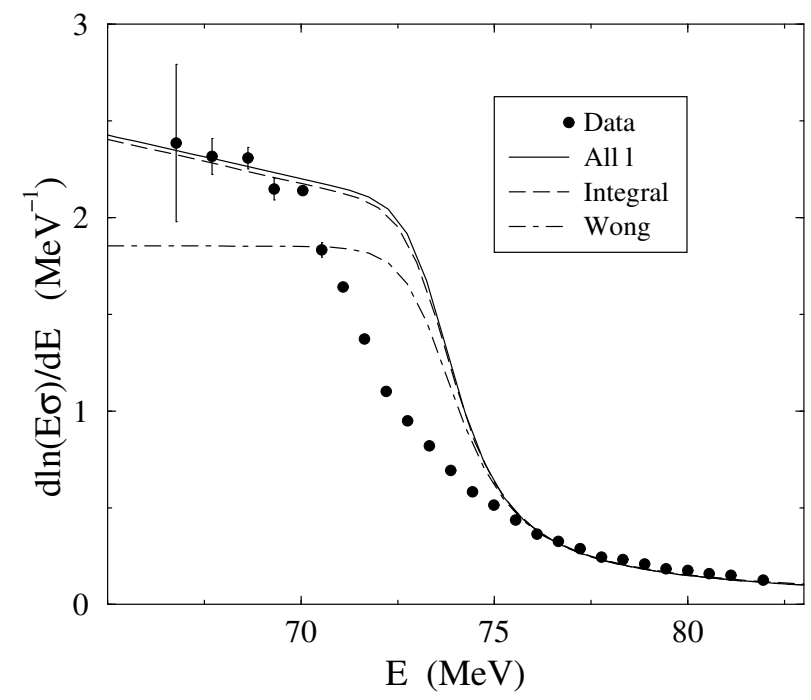

FIG. 7: The expression (11) reproduces very well the logarithmic derivative far below the Coulomb barrier. This is an uncoupled calculation for ${ }^{16} \mathrm{O}+{ }^{208} \mathrm{~Pb}$ with $a=1.65 \mathrm{fm}$. The Wong approximation does not reproduce the correct behavior in this energy region.

We do not attempt to provide an explanation of this phenomenon but merely wish to show here that an integral expression is capable of reproducing the cross section sufficiently well at these low energies if one wishes to study this property. Using Eq. (11), we may write for a single barrier

$$
\frac{d \ln (E \sigma)}{d E}=\frac{d}{d E} \ln \int_{0}^{E} T_{0}\left(E^{\prime}\right) d E^{\prime} \equiv \frac{T_{0}(E)}{\int_{0}^{E} T_{0}\left(E^{\prime}\right) d E^{\prime}} .
$$

Fig. 7 reproduces the Fig. 7 of Dasgupta from Ref. [21] for a single uncoupled barrier in ${ }^{16} \mathrm{O}+{ }^{208} \mathrm{~Pb}$. There is no attempt here to fit the data except the logarithmic derivative at the lowest energies. We see that the results from the integral formalism again agree extremely well with a calculation including all $l$ values. The Wong approximation, however, fails in this region. One may easily show from Eq. (13) that the Wong value saturates at $2 \pi / \hbar \omega$, which is $1.85 \mathrm{MeV}^{-1}$ in this case.

For a single potential barrier, certain analytic expressions exist within the WKB approximation which allow the inversion of the barrier penetration to yield the barrier thickness, and thus the form of the potential itself [22]. Early applications [23] of this technique to heavy-ion fusion cross sections yielded rather unphysical, often multi-valued potentials, since the cross section actually comes from a distribution of barriers. It has very recently [24] been shown, however, that in certain circumstances (essentially where the lowest barrier is dominant), one may take account of this fact and perform the inversion more correctly. In particular the above case of ${ }^{16} \mathrm{O}+{ }^{208} \mathrm{~Pb}$ is susceptible to such a treatment. The potential thus obtained is well behaved but rather different from most 'standard' heavy-ion potentials. It remains to be seen if a theoretical justification for the new shape can be found, or whether the potential (albeit well behaved) still mocks up some other, missing, physical effect.

\section{OTHER PHYSICAL PROPERTIES}

\section{A. General comments}

We have seen how the total capture cross section

$$
\sigma=\frac{\pi}{k^{2}} \sum_{l=0}^{\infty}(2 l+1) T_{l}
$$

can be approximately represented in terms of an integral containing simply the transmission coefficient $T_{0}^{\text {tot }}$ for the s-wave. Eq. (16) contains a single integral over $E^{\prime}$ which replaces the above sum over the partial waves. Eq. (19), however, contains a second integral over $B$ which formally represents a sum over different Coulomb barriers.

These results can be simply extended to other physical quantities such as the fusion-fission cross section, the quasi-fission cross section and the fusion-evaporation cross section. For example the quasi-fission cross section may be written

$$
\sigma_{\mathrm{QF}}=\frac{\pi}{k^{2}} \sum_{l, \alpha}(2 l+1) w_{\alpha} T_{l}\left(E, B_{\alpha}\right) P_{\mathrm{QF}}(l, E, \alpha),
$$


This can also be appreciated from the rotational calculation of Fig. 6, where the overlapping barriers also wash out the individual peaks. However, having obtained the relevant values one can use them to generate the full cross section, which is essentially indistinguishable from the solid curve in Fig. 4 a. We might, therefore, expect that the corresponding expressions:

$$
(2 l+1) T_{l} \approx(2 l+1) \sum_{\alpha} w_{\alpha} T_{l}\left(E, B_{\alpha}\right)
$$

will give a reasonable approximation to the spin distribution at an energy $E$.

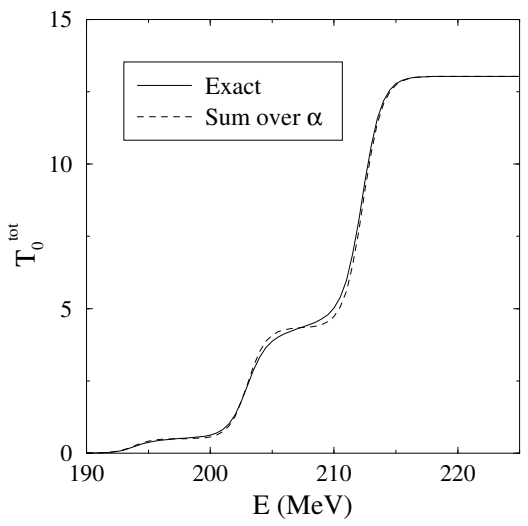

FIG. 12: The exact $T_{0}^{\text {tot }}$ (solid line) which corresponds to the system of Fig. 4, along with the sum of uncoupled calculations (dashed line) with the barrier weights and heights $\left(w_{\alpha}, B_{\alpha}\right)=$ $(0.038,193.80) ;(0.295,202.96)$ and $(0.667,212.30)$.

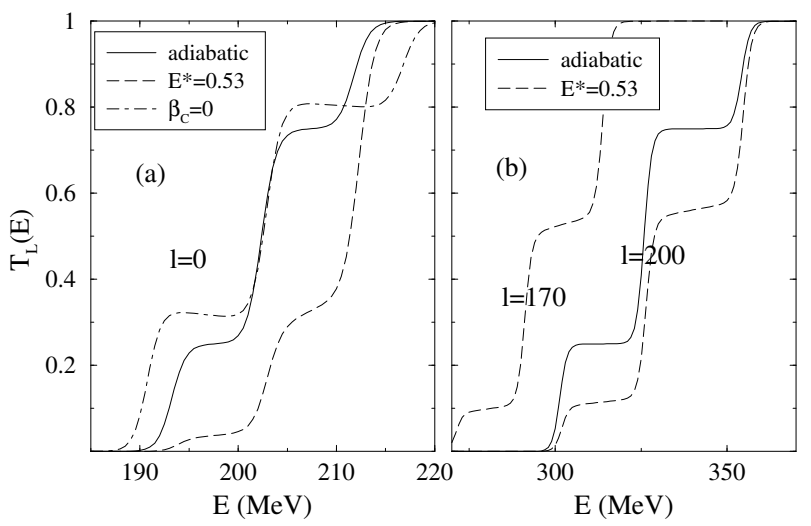

FIG. 13: (a) For a quadrupole phonon with $E^{*}=0.53 \mathrm{MeV}$, $T_{0}^{\text {tot }}$ (dashed line) is very different from its adiabatic value (solid line) for $l=0$, in particular the weight of the lowest barrier is greatly reduced. With no Coulomb coupling (dotdashed line), however, the lowest barrier is actually enhanced relative to the adiabatic value. (b) For higher $l$, the barrier occurs at higher energy where the weights come closer to their adiabatic values. Calculations for $l=170$ and $200 \hbar$ show that the weights vary little over a limited range of angular momenta/energy.

Here, we could use the parabolic approximation for the
$T_{l}\left(E, B_{\alpha}\right)$, employing appropriate values of $R_{\alpha}$ and $\omega_{\alpha}$. Alternatively, we could calculate the $T_{l}\left(E, B_{\alpha}\right)$ directly with uncoupled potentials having the same diffuseness $a$ as the original calculation, since this will reproduce more correctly the properties of each barrier (for example the variation with $l$ ). We choose to do the latter and the results of this procedure are shown by the dashed lines in Fig. 11. We see that while the positions of the steps are reproduced reasonably well, the heights of the steps are incorrect at higher energies. In other words the barrier weights appear to change as a function of the incident energy. Although this has a marked effect on the spin distribution, it has little effect on the total cross section which is summed over $l$.

It is clear from Fig. 12 that the weights and heights of the barrier distribution can be readily obtained from the steps in $T_{0}^{\text {tot }}[33]$. The $B_{\alpha}$ occur where $T_{0}^{\text {tot }}$ rises most steeply, and the $w_{\alpha}$ are given by the differences between the steps in the function. (As noted above, this is less clear for smoother distributions.) How then can we define the weights at a higher energy, where $T_{0}$ has already become 1? The answer is to look at the same function but for a higher $l$ value, for which the total potential barrier will occur around the energy in question.

This is demonstrated in Fig. 13 for the above system. Fig. 13 a shows $T_{0}^{\text {tot }}$ calculated for the physical excitation energy of $0.53 \mathrm{MeV}$ (dashed curve). One sees that the weights are very different from their adiabatic values $\left(E^{*}=0 ;\right.$ solid curve). If one calculates the same function for an octupole phonon with the same $E^{*}$ and $\beta$ (not shown), the weights are close to their adiabatic values. The major difference between these two cases is the importance of the Coulomb coupling at large distances. In the quadrupole case, the range is long enough for the Coulomb coupling to strongly polarize the entrance channel before the barrier is reached [32]; clearly this coupling will favour the barrier for which the Coulomb field is lowest, that is, the highest of the barriers. This assertion can be confirmed by performing a calculation where the Coulomb coupling is switched off. This is shown by the dot-dash curve in Fig. 13 a. Now we see that the weight of the lowest barrier is actually enhanced (due to the nuclear couplings) relative to the adiabatic value.

In Fig. $13 \mathrm{~b}$, we show $T_{l}$ for $l=200$, for which the barrier in the total potential (including the centrifugal term) occurs at around $325 \mathrm{MeV}$. The steps now give the barrier weights in this energy region. They are seen to be closer to their adiabatic values but still far from converged to these. We also show here the same quantity for $l=170$ and see that the non-adiabatic weights do not vary significantly over the corresponding energy range. The reason for the slow convergence of the weights is that although the timescale associated with a $0.53 \mathrm{MeV}$ excitation is relatively long, the long range of the quadrupole Coulomb field gives sufficient time for a strong polarization to take place even with an incident energy of more than $300 \mathrm{MeV}$.

A further interesting feature of Fig. $13 \mathrm{~b}$ is that the 
energy difference between the highest and lowest barriers is significantly larger for $l=200$ than for $l=0$; around $53 \mathrm{MeV}$ compared with $18 \mathrm{MeV}$. The reason for this is that the barriers for high $l$ occur at a smaller radius than for $l=0$, and in this region the nuclear coupling form factor is correspondingly larger. We note that this effect is specifically $l$-dependent rather than $E$-dependent.

The variation of the quadrupole Coulomb polarization is the origin of the discrepancies seen in the spin populations calculated with fixed weights in Fig. 11. We note that variations of the weights that we report here are larger than those discussed in Ref. [33]. However, there is no contradiction with these results, since the authors of that paper did not consider the long-ranged quadrupole Coulomb couplings and did not, in any case, study such a large energy range as here.

At energies near the $l=0$ barrier, the quadrupole Coulomb field is the origin of the entrance-channel 'extrapush' energy described in Ref. [12], where the energy at which $T_{0}^{\text {tot }}=0.5$ is increased by this polarization. For the present simplified [1,1] coupling scheme the shift is around $E_{\mathrm{x}}=9 \mathrm{MeV}$ (see Fig. 13 a) relative to the uncoupled barrier (a higher value is produced if multiplephonon states are included in the coupling), significantly reducing the anomaly between the Bass barrier and the dynamical barrier observed in many symmetric heavy-ion reactions [12].

\section{CONCLUSIONS}

We have presented new results relating to nuclear reactions which are governed by strong couplings in the entrance channel and, therefore, to the existence of a distribution of barriers. In some cases these effects can be easily incorporated into calculations of quasi-fission and evaporation-residue cross sections. This was achieved without the need to extract the heights and weights of the corresponding barrier distribution but simply by exploit- ing the the $s$-wave transmission coming from a standard coupled-channels calculation.

The only occasion on which we explicitly introduced barrier weights was to show that they may change with incident energy. But even in this case, the effect is important only for quadrupole-phonon states, only at energies high above the barrier and only in calculating the spin distribution of the compound nucleus rather than cross sections, which are summed over $l$. However, even at near-barrier energies, highly collective target and projectile quadrupole-phonon states can give rise to an important extra-push energy, that is, a significant shift of the average (dynamical) barrier to higher energies.

The reaction ${ }^{48} \mathrm{Ca}+{ }^{154} \mathrm{Sm}$ was studied in some detail and, from a single $T_{0}^{\text {tot }}$, good results were obtained for the capture, quasi-fission and evaporation-residue cross sections, all of which been measured in this system. While the functions coupled with $T_{0}^{\text {tot }}$ to give the ER cross section, have a good theoretical basis in the statistical-decay model, the function used to describe the quasi-fission was simply a generalization of the 'compactness' parametrization suggested in Ref. [17]. The data studied strongly suggest the correctness of this notion. It would, therefore, be good to have a theoretical model for this effect, especially since it appears to play a major role in systems leading to super-heavy element creation by hot fusion [34].

\section{Acknowledgments}

One of the authors (NG) is grateful to the INFN Laboratori Nazionale di Legnaro, in particular to Prof. Alberto Stefanini, for a fruitful visit during which this work was started. She is also grateful to the IPHC, Strasbourg, in particular to Dr. Johann Bartel, for several visits during which the present work and her thesis were completed.
[1] S.S. Ntshangese et al., Phys. Lett. B651, 27 (2007).

[2] H. Ikezoe et al., in Proc. of FUSION06, Venezia, Italy 1923 March 2006, Eds. L. Corradi et al., AIP Conference Proceedings 853, 69 (AIP, New York, 2006)

[3] K. Hagino and N. Rowley, Phys. Rev. C 69, 054610 (2004).

[4] N. Rowley, G.R. Satchler and P.H. Stelson, Phys. Lett. B254, 25 (1991).

[5] M. Dasgupta, D.J. Hinde, N. Rowley, and A.M. Stefanini, Ann. Rev. Nucl. Part. Sci. 48, 401 (1998).

[6] H. Timmers et al., Nucl. Phys. A633, 421 (1998); A.M. Stefanini et al., Phys. Rev. C 76, 014610 (2007).

[7] A.B. Balantekin and N. Takigawa, Rev. Mod. Phys. 70, 77 (1998).

[8] L.F.Canto, P.R.S.Gomes, R.Donangelo, M.S.Hussein, Phys. Rep. 424, 1 (2006).

[9] A.B. Quint et al., Z. Phys. A346, 119 (1993).
[10] W. Reisdorf, Z. Phys. A300, 227 (1981); W. Reisdorf and M. Schädel, Z. Phys. A343, 47 (1992).

[11] R. Bass, Phys. Rev. Lett. 39,265 (1977).

[12] N. Rowley, N. Grar and K. Hagino, Phys. Lett. B632, 243 (2006).

[13] S. Bjornholm, W.J. Swiatecki, Nucl. Phys. A391, 471 (1982); J.P. Blocki, H. Feldmeier and W.J. Swiatecki, Nucl. Phys. A459, 145 (1986).

[14] C.Y. Wong, Phys. Rev. Lett. 31, 766 (1973).

[15] K. Hagino, N. Rowley and M. Dasgupta, Phys. Rev. C 67, 054603 (2003).

[16] N. Rowley, A. Kabir and R. Lindsay, J. Phys. G15, L269 (1989).

[17] D.J.Hinde et al., Phys. Rev. Lett. 74, 1295 (1995).

[18] K. Hagino, N. Rowley and A.T. Kruppa, Comp. Phys. Comm. 123, 143 (1999).

[19] C.L. Jiang, B.B. Back, H. Esbensen, R.V.F. Janssens, 
K.E. Rehm, Phys. Rev. C 73, 014613 (2006).

[20] H. Esbensen, Prog. Theor. Phys. (Kyoto), Suppl. 154, 11 (2004).

[21] M. Dasgupta, in Proc. of FUSION06, Venezia, Italy 1923 March 2006, Eds. L. Corradi et al., AIP Conference Proceedings 853, 21 (AIP, New York, 2006).

[22] M.W. Cole and R.H. Good, Phys. Rev. A18, 1085 (1978).

[23] A.B. Balantekin, S.E. Koonin and J.W. Negele, Phys. Rev. C 28, 1565 (1983).

[24] K. Hagino and Y. Watanabe, Phys. Rev. C, Rapid Communications, in press.

[25] A. M. Stefanini et al., Eur. Phys. J. A23, 473 (2005); M. Trotta et al., Eur. Phys. J. A25, 615 (2005).

[26] G.N. Knyazheva et al., Phys. Rev. C 75, 064602 (2007).
[27] G.G. Adamian, N.V. Antonenko, W. Scheid and V.V. Volkov, Nucl. Phys. A627, 361 (1997) and references therein.

[28] J. Toke and W.J. Swiatecki, Nucl. Phys. A372, 50 (1981).

[29] S. Cohen, F. Plasil and W.J. Swiatecki, Ann. Phys. (NY) 82, 557 (1974).

[30] N. Grar and N. Rowley, in progress

[31] B Herskind et al., Phys. Scr. T125, 108 (2006).

[32] A.S. Jensen and C.Y. Wong, Phys. Rev. C 1, 1321 (1969).

[33] K. Hagino, N. Takigawa and A.B. Balantekin, Phys. Rev. C 56, 2104 (1997).

[34] Yu.Oganessian, J.Phys.(London) G34 (2007) R165. 